\title{
Los límites de los recursos electrónicos en la democracia deliberativa
}

\author{
The Limits of Electronic Resources \\ in Deliberative Democracy
}

\author{
FEDERICO G. ABAL \\ Universidad de Buenos Aires - GFP \\ Recibido: 08/II/2014 Aceptado definitivamente: 27/VII/2014
}

\begin{abstract}
RESUMEN
Las redes sociales han tenido un papel importante en el desarrollo de reclamos cívicos recientes. Internet presenta rasgos favorables para un libre intercambio de razones entre ciudadanos y ofrece un espacio nuevo para la circulación de información. En el presente trabajo me propongo indagar sobre el alcance de los recursos electrónicos y su vinculación con las concepciones deliberativas de la democracia.

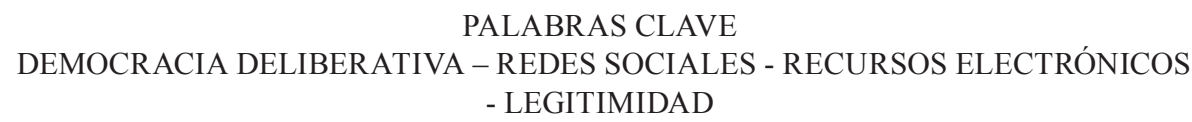

Social networks have played an important role in the development of recent civic complaints. Internet has favorable features for a free exchange of reasons between citizens and provides a new space for the circulation of information. This paper investigates the scope of electronic resources and its relationship to deliberative conceptions of democracy.

\section{KEYWORDS}

DELIBERATIVE DEMOCRACY - SOCIAL NETWORKS - ELECTRONIC RESOURCES - LEGITIMACY

(C) Contrastes. Revista Internacional de Filosofia, vol. XX No 2 (2015), pp. 201-216. ISSN: 1136-4076 


\section{INTRODUCCIÓN}

ENTRANDO EN LA SEGUNDA DÉCADA DEL SIGLO XXI, podemos observar una profunda y extendida crisis de legitimidad y representación en los más variados puntos del mundo. Prueba de ello son los movimientos de indignados en España, los manifestantes de Ocuppy Wall Street, las recientes protestas en Kiev, las expresiones de la Primavera Árabe y los conflictos institucionales que azotan a varios países de América latina.

La consigna que parece reproducirse a lo ancho y largo del globo es «queremos democracia». Hay una fuerte percepción por parte de la ciudadanía de que los sistemas democráticos vigentes reconocen a los ciudadanos, sencillamente, como miembros de una turba incapaz de autogobernarse a los cuales solo les compete la tarea de ocasionalmente afirmar en el poder a las distintas elites partidarias que monopolizan la toma de decisiones políticas.

Aunque los recientes reclamos cívicos por la democratización del poder político han emergido generalmente en situaciones de profundas crisis económicas, no necesariamente debe llegarse a elevados índices de desocupación, marginalidad o flagrantes violaciones a los derechos humanos para que los ciudadanos se convoquen con dicho propósito. El caso de las movilizaciones que tuvieron lugar recientemente en Frankfurt resulta interesante en este punto, porque presenta un importante grado de participación (entre estudiantes, trabajadores y jubilados) en el marco de una economía desarrollada y con altos niveles de calidad educativa, laboral y sanitaria. ${ }^{1}$

Los avances de Internet han cumplido un rol fundamental en este tipo de movilizaciones. Las redes sociales, los blogs y los foros han permitido un espacio de intercambio constante que en muchos casos decantó en convocatorias concretas y multitudinarias. La aparición de los denominados partidos piratas, con una apreciable intervención electoral (obteniendo, por ejemplo, un 9,1\% en Republica Checa, 7,13\% en Suecia y 9,0\% en Ucrania), da cuenta de la importancia de las nuevas tecnologías en la vida política y las propuestas que pueden ofrecerse en base a ellas.

Este panorama fortaleció una tendencia que, desde mediados del 2000, tiene lugar en la filosofía política contemporánea. Algunos teóricos interesados en las concepciones deliberativas de la democracia encuentran en internet un marco adecuado para llevar adelante los rasgos centrales de esta alternativa (Dahlberg 2001, Marti y Canals 2003, Robles y Ganuza 2011, entre otros). En el presente trabajo, me propongo evaluar las conexiones de las propuestas de

1 Ver: < http://www.clarin.com/mundo/Marcha-Alemania-ajuste-bancos_0_703729715. html $>(20 / 05 / 2012)$ o $<$ http://www.lagaceta.com.ar/nota/547115/mundo/protestas-contra-politica-neoliberal-union-europea.html $>(2 / 06 / 2013)$. 
democracia electrónica con las formulaciones estándar de la democracia deliberativa. Luego, busco rastrear ciertos límites del uso de los recursos electrónicos y sus posibles alcances en ámbitos que presentan características especiales.

\section{Democracia Deliberativa y E- Democracia}

Frente a una concepción conservadora, mercantilista y oligárquica de la vida política, bastante extendida, la democracia deliberativa emerge como una alternativa que considera el razonamiento público como fuente de legitimidad de las decisiones políticas vinculantes.

Si bien los teóricos de la democracia deliberativa difieren en varios aspectos unos de otros, creo que puede ofrecerse una presentación estándar que rescate aquellos rasgos de la concepción que parecen ser compartidos por las expresiones más destacadas (por ejemplo, Bohman 1996, Habermas 1999 y Elster 2001). ${ }^{2}$ Podríamos definirla como una propuesta que caracteriza al debate público como el espacio propio para la búsqueda de consensos sobre el bien común, la transformación de preferencias y la maximización del bienestar general, entendido como el resultado de un acuerdo razonable, libre y revisable.

La deliberación se funda en el reconocimiento de las personas como libres e iguales; esto es, como sujetos capaces de adoptar una determinada filosofía de vida y con derecho de tener igual influencia en la toma de decisiones que les afectan. ${ }^{3}$

Al cuestionar no solo el elitismo (que supone la existencia de personas mejor capacitadas para ejercer la autoridad política, ya sea por su posición económica o su capacidad cognitiva), sino también el mayoritarismo (que afirma que la legitimidad de las decisiones se funda únicamente en la regla de la mayoría, entendida como la agregación de preferencias individuales o sectoriales) como interpretaciones de la democracia, la deliberación se compromete con una noción de reciprocidad. La reciprocidad remite a un compromiso que les corresponde asumir a los ciudadanos de una comunidad democrática y que los compele a evitar las imposiciones arbitrarias sobre los demás, ofreciendo razones para sus acciones políticamente relevantes (voto, iniciativas, reclamos,

2 Para un acercamiento a distintas concepciones de la deliberación, ver: J. Montero (2005) «¿Qué criterio de igualdad requiere una democracia deliberativa?» en Diánoia, volumen L, número 55, noviembre, pp. 119-135 y Oquendo, A. (2005) «Más allá de la democracia deliberativa» en Polis, 10.

3 Entiendo por filosofía de vida, lo que J. Cohen denomina de ese modo; a saber, «una perspectiva total, abarcadora (religiosa o laica, liberal o tradicionalista) que incluya un catalogo de todos los valores éticos y proporcione una guía general a la conducta, tanto la individual como la colectiva» (Cohen, 2001, 238).. 
etc.) que los participantes pueden reconocer como tales. ${ }^{4}$

El desarrollo de internet, la emergencia de las redes sociales y su vinculación real con la práctica política, ofrecen un campo interesante para indagar respecto de los servicios que podrían prestar en el mejoramiento del razonamiento público.

En primer lugar, debemos decir que las opciones democráticas que proponen el aprovechamiento de estas nuevas tecnologías guardan una relación estrecha con las motivaciones que pueden rastrearse en los teóricos de la deliberación.

Los modelos de E-democracia que reivindican los partidos piratas surgen de una lectura pesimista de los sistemas políticos vigentes. ${ }^{5}$ Frente a la exclusión de la ciudadanía de las decisiones que le afectan y el anquilosamiento de estructuras partidarias que no responden a distintas demandas ciudadanas, la respuesta más clara de ampliar los canales de participación parece encontrar eco en el mundo virtual.

Si nos remitimos al nivel de influencia de internet en nuestra vida cotidiana y su aparente constitución como ágora contemporáneo, la relación con la democracia deliberativa es fácilmente trazable. Tal como señala Marti, las nuevas tecnologías tienen un gran potencial para facilitar los ideales que persigue la deliberación. ${ }^{6}$

Hay dos rasgos de internet que pueden señalarse en este sentido. En primer lugar, constituye una red, en apariencia, distribuida; esto es, compuesta por nodos con una capacidad de influencia y distribución de información equitativa. La posibilidad que tenemos de escribir un blog, discutir en un foro, subir videos o grabar un discurso parece afirmar esta condición.

4 Por «razón» me refiero a la idea de «consideración a favor de algo». Es tal como explica Scanlon, una noción primitiva al uso mismo de la racionalidad humana. Una razón para algo es una consideración que cuenta a su favor. Dado el hecho concreto de que las personas se encuentran divididas por doctrinas comprehensivas incompatibles, es de esperar que las razones fundadas en consideraciones religiosas o sectoriales no sean reconocidas como tales. Ver: T.M Scanlon (1998) Cap 1: Reasons de What we owe to each other. Cambridge: Harvard UP.

5 Ver, entre otros: < http://partidopirata.com.ar/ >, < http://confederacionpirata.org/ $>,<$ http://www.pp-international.net/ $>\mathrm{y}<\mathrm{http}: / /$ uspirates.org/ $>$.

6 Link: < http://www.lavanguardia.com/cultura/20120418/54284555764/marti-mecanismos-democracia-mas-ambiciosa.html $>(18 / 04 / 2012)$. 


\section{Red Centralizada Red Descentralizada Red Distribuida}
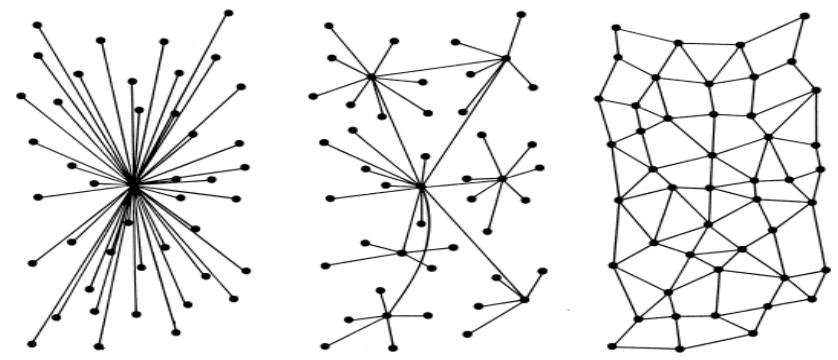

Desde la teoría de redes de Barabasi (1999, p. 509), podemos afirmar que Internet es también una red autoorganizada o libre de escala. Una estructura compuesta de nodos y enlaces distribuidos de forma despareja, donde no hay nodos preexistentes sino provisionales y como consecuencia imprevisible de la actividad de los agentes. Estas dos características (distribución y autoorganizacion), han llevado a considerar a Internet, con sus respectivos avances, como el espacio adecuado para tratar la legitimidad de las decisiones políticas. ${ }^{7}$

Las alcances de este mundo virtual que se nos ha abierto son difíciles de precisar, debido a su vertiginoso desarrollo; sin embargo, los acontecimientos internacionales recientes que mencionamos al comienzo, y que tienen un denominador común en el uso de las redes sociales y los dispositivos interactivos, nos exigen que reconozcamos ciertos rasgos que llevan a la ciudadanía a convocarse en ellos. Porras (2005) ha señalado claramente las posibilidades que se siguen de la distribución y autoorganizacion de la red.

«Los dispositivos interactivos y multifuncionales de las tecnologías recientes nos permiten eludir el control que determinados actores públicos y privados ejercen en la producción y gestión de la comunicación política, y hacen plausible pensar en una arena conversacional en la que: a) todos los actores pueden ejercer indistintamente como emisores o receptores; b) se produzca una ilimitada cantidad y variedad de informaciones, sin otras restricciones que las que se autoimpongan sus emisores; $y$ c) puedan darse formas de conexión colectiva en tiempo real $y$, por tanto, la formación de flujos discursivos.» (pp. 105 - 106)

7 William Dutton, por ejemplo, señala que las redes sociales se constituyen actualmente como un «Fifth Estate», que se aparta de las formas tradicionales del ejercicio político y que desafía la institucionalidad establecida. Ver: Dutton, W. H. (2009) «The Fifth Estate Emerging trough the Network of Networks» en Prometheus: Critical Studies in Innovation, 27/1. pp. 1-15. 


\section{Problemas de IMPlementación}

La Comisión Europea define a la E- democracia o democracia electrónica como «el uso de las tecnologías de la información y las comunicaciones en las administraciones públicas, combinado con cambios organizativos y nuevas aptitudes con el fin de mejorar los servicios públicos, los procesos democráticos y reforzar el apoyo a las políticas públicas». ${ }^{8}$

En primer lugar, vale decir que no parece ser interesante discutir respecto de la utilización de las nuevas tecnologías para mejorar nuestras democracias. La amplia mayoría de los teóricos preocupados por los índices de transparencia, el acceso a la información y la participación política están de acuerdo en que podemos valernos de los actuales soportes tecnológicos para llevarlo adelante (mediante encuestas online, foros de discusión o blogs donde compartir las últimas novedades).

La cuestión que me propongo abordar, por el contrario, ha admitido distintas posiciones y se refiere a la conexión directa entre democracia deliberativa y E-democracia. Me pregunto si efectivamente internet nos brinda un espacio apto para desarrollar los valores implícitos en esa tradición o si reproduce ciertos problemas propios de las instancias de toma de decisión vigentes.

Como todo fenómeno importante, el desarrollo de las nuevas tecnologías ha generado diferentes lecturas. Siguiendo la línea de Garot (2006), podemos marcar posiciones optimistas y pesimistas respecto a las posibilidades que ofrece Internet.

Los primeros consideran las redes sociales, los blogs, los foros, los chats y los canales de videos como la manera más adecuada para vincular a los ciudadanos en una democracia directa, deliberativa y fuerte. ${ }^{9}$ Ya he mencionado que, experiencias recientes como las movilizaciones de indignados en España y la Primavera Arabe, parecen resaltar esta posibilidad al mostrar ciudadanos que libremente se encuentran en las redes sociales y consiguen formular un reclamo común.

Las interpretaciones más pesimistas suelen destacar problemas estructurales que no parecen afectar la validez de las alternativas de E-democracia. Las críticas que apuntan a las desigualdades en el acceso a internet vinculadas

8 Comunicación de la Comisión al Consejo, al Parlamento Europeo, al Comité Económico y Social Europeo y al Comité de las Regiones, El papel de la administración electrónica en el futuro de Europa, Bruselas, COM (2003), 567 final, 26.9.2003, p 7

9 En esta dirección, Pierre Levy señala que «Internet abre un nuevo espacio de comunicación, inclusivo, transparente y universal, que está llamado a renovar profundamente las condiciones de la vida pública en el sentido de una libertad y de una responsabilidad mayores de los ciudadanos.» Etude sur la gouvernance européenne et la cyberdemocratie, Comisión Europea, Bruselas, 30/1/2001, p. 38 
con profundas inequidades sociales para señalar un límite a estas propuestas no parecen ser concluyentes desde un punto de vista filosófico.

El presente trabajo propone una línea más cercana a las investigaciones de Norris y Curtice (2004) sobre los desafíos internos de estos modelos. En este sentido, creo que los problemas más interesantes de la E- democracia, son los que refieren a sus fundamentos teóricos y sus límites para plantear soluciones a los desafíos democráticos actuales. En esta dirección, sugiero distinguir entre dos clases de problemas: (i) de implementación y (ii) teóricos.

Por problemas de implementación entiendo inconvenientes de orden práctico que podrían ser resueltos mediante los avances de los soportes electrónicos, el mejoramiento de los foros de discusión y las instancias de elección online, y el trabajo sostenido de programadores con capacidad imaginativa. Entre ellos, distingo los siguientes, rastreables en la actual etapa de desarrollo del software.

\section{III.1 LA ORGANIZACIÓN DE LA PARTICIPACIÓN CIUDADANA}

Uno de los rasgos fundamentales de la democracia deliberativa es el libre intercambio de razones. Sería absurdo pensar en diseños institucionales que permitieran la participación argumentativa de absolutamente todos los ciudadanos en la mayoría de los temas. Es probable que una porción de la ciudadanía no se sienta interpelada por las discusiones políticas y prefiera reducir su actividad a las instancias electorales o reclamos muy puntuales que le afecten particularmente. ${ }^{10}$

Lo que se pretende garantizar es la inclusión de las distintas justificaciones públicas. En gran medida éstas no son individuales y se enmarcan en reclamos o propuestas partidarias, sindicales, de minorías sexuales o de organismos de derechos humanos. La deliberación asume un filtro que permite el ingreso al razonamiento público de aquellas razones que son atendibles. Por supuesto que dicho filtro debe ser lo más plural, democrático e inclusivo posible. ${ }^{11}$

Los defensores de la democracia electrónica suelen proponer soportes

10 Esta situación se produjo en la UK Citizens Online Democracy. Ver: Dahlberg, L. «The Internet and democratic discourse» en Information, Communication and Society 4: 4, (2001) p. 615-633 y < www.mysociety.org/files/2011/06/TheyWorkForYou_research_report-2011-Tobias-Escher1.pdf $>(05 / 2012)$.

11 Esto muestra el nivel de exigencia del ideal deliberativo en términos de reformas institucionales y sociales. Entre otras cosas exigiría una democratización de las instancias de participación política (partidos, sindicatos, etc.) y una ley que regule la concentración mediática. Para un acercamiento a las exigencias de la democracia deliberativa, ver: Fung, A. (2005) «Deliberation before the Revolution: Towards an ethics of deliberative democracy in an unjust world» en Political Theory, Vol. 33, No. 3, Junio, pp. 397 - 419. 
virtuales donde los ciudadanos podrían informarse, debatir y votar. Es probable que en esa libre participación, alcancemos largas cadenas de comentarios, indexadas según algún ranking de votación, lo que volvería dificultoso el intercambio de razones propio de las concepciones deliberativas de la democracia. Basta solo echar un vistazo a la infinidad de foros que circulan por la Red y la imposible tarea de seguir todas las intervenciones de los participantes. Muchos espacios de investigación y escuelas de políticas públicas proponen medidas para evitar este tipo de situaciones. ${ }^{12}$ Una de ellas es limitar el número de comentarios posibles por persona (como en la iniciativa de E-democracia en Minnesota), otro es participar del debate cediendo nuestra voz y voto a otros usuarios que reconocemos con un mayor grado de experticia sobre lo que se está discutiendo. ${ }^{13}$

\section{2 CORPORACIONES Y HUBS}

La horizontalidad que plantean las redes sociales e Internet en general ha llevado a considerar este espacio como una esfera libre de dominio externo. La libre circulación de información públicamente relevante es indispensable para cualquier comunidad que se precie de ser democrática.

Los distintos conglomerados empresariales-mediáticos generalmente vinculados con la administración política de turno ponen límites inmorales al desenvolvimiento de la información; invisibilizando los reclamos de las minorías, impidiendo la difusión de medios más pequeños, encubriendo casos de corrupción y conexiones con el poder político a cambio de favores en la distribución de licencias, entre otros hechos conocidos en muchos países. Ante ello, Internet presenta una posibilidad única de divulgación por fuera de los caminos marcados por el aparato oficial y el establishment privado.

Sin embargo, podemos permitirnos dudar de esta afirmación dado el mapa actual de la Red. Grandes corporaciones aparecen como dominadores de los espacios de distribución de información: Google, Facebook, YouTube.

Eben Moglen (2012) ha señalado el desafío que presentan estos emporios virtuales a la libertad de pensamiento y el control de nuestros datos personales. ${ }^{14}$ Jullian Assange, fundador de Wikileaks, ha denunciado las vinculaciones entre el Departamento de Estado de los EE.UU y Jared Cohen, director del

12 Por ejemplo: $<$ www.egov.vic.gov.au $>$, $<$ www.edemocracycentre.ch $>,<$ www.c2d. $\mathrm{ch}>\mathrm{y}<$ www.edemocracia.net $>$.

13 Esta iniciativa es propuesta por el Partido de la Red (Argentina), un espacio nuevo inspirado en los partidos piratas europeos. Ver: $<$ http://partidodelared.org/democracia-os/ $>$

14 Ver la conferencia de Moglen en Re:Publica (Berlin, Mayo, 2012) Link: < http://www. youtube.com/watch? $\mathrm{v}=\mathrm{HJCczbSF}-\mathrm{B} 8>(1 / 6 / 2012)$. 
centro Google Ideas. ${ }^{15}$

Dentro de la Teoría de Redes, los nodos que tienen una enorme capacidad para difundir o filtrar datos se denominan hubs. Si bien, la posibilidad de una distribución simétrica de oportunidades para hacer circular información es poco habitual, ya que las características de los agentes llevan a desajustes y distintos niveles de almacenamiento de data, la existencia de nodos jerárquicamente superiores (por ejemplo, navegadores y administradores de redes sociales) sin ningún tipo de control por parte de los demás podría traducirse en casos de censura o manipulación de nuestros datos personales, indeseables para un modelo democrático de participación. ${ }^{16}$

Los distintos desarrollos del software libre y la difusión de los principios hackers entre los programadores del mundo son el medio más promisorio para alcanzar redes mas distribuidas.

\section{3 NETOCRACIA Y EL RIESGO DE LOS BAD DIGERATI}

Netocracia es un término acuñado por los infonomistas suecos Jan Soderqvist y Alexander Bard (2003). Con él se proponen describir lo que consideran la nueva clase emergente del naciente mundo postcapitalista o informacionalista. Los netócratas conforman una elite con un alto grado de preparación en materia de medios y programación, y se encargan de intervenir la Red imponiendo un determinado direccionamiento de las preferencias individuales de cada usuario.

En un mundo que no ha resuelto las necesidades más urgentes de una importante parte de su población (de salud, alimentación y vivienda), la brecha digital (la inmensa mayoría de la población mundial que no tiene acceso a Internet) y la divulgación de los conocimientos básicos de programación parecen cuestiones poco relevantes. Sin embargo, tal como afirma Peña-Lopez (2011, p. 14) la influencia de las nuevas tecnologías es tan avasallante que los diseños institucionales nacionales actuales no pueden generar dispositivos de control sobre ciertos agentes virtuales supranacionales que afectan directa o indirectamente a nuestra vida cívica.

La búsqueda por ampliar la democracia, volverla más participativa y

15 Link: < http://m.telesurtv.net/articulos/2013/08/25/julian-assange-google-trabaja-atado-a-ee.uu-2868.html >.

16 Sobre la asimetría en la centralidad de los nodos, ver: Centola, D. (2010) «The Spread of Behaviour in an Online Social Network Experiment» en Science, 329, 4, n 5996, pp. 1194-1197 $y$ Liu, Y, Slotine, J.J. , Barabasi, A.L. (2012) «Control Centrality and Hierarchical Structure in Complex Networks» en Plos One, Septiembre, Vol. 7, Issue 9. 
transparente, del modo en que proponen los modelos electrónicos, parece permitir la entrada al juego político de una nueva casta capaz de manipular información, intervenir soportes electorales y rediseñar el mundo virtual para su propio beneficio. ${ }^{17}$ Esta clase de netocratas, Peña-Lopez los denomina bad digerati y establecen un problema muy claro: si las nuevas tecnologías se nos presentan a la mayoría como un gran enigma, lleno de posibles operaciones netocraticas perjudiciales, parece más sensato plantear reformas profundas en nuestras democracias actuales, que se muestran exentas de un innovación constante en sus requisitos técnicos.

\section{Problemas teóRICos}

Los problemas que denomino teóricos son internos a los modelos de Edemocracia, no parecen resolubles mediante un mayor desarrollo de las nuevas tecnologías, se alejan de la concepción de la democracia deliberativa y parecen reproducir ciertos inconvenientes que encontramos en las instancias vigentes de toma de decisiones.

\section{IV.1 DÉFICIT DE RAZONABILIDAD}

Ya he mencionado que en las formulaciones más tradicionales de la democracia deliberativa parece asumirse un criterio de razonabilidad para la instauración de determinados temas en la agenda del debate público (Benhabib 1999, Guariglia 2002, p. 153). Los reclamos y las propuestas fundadas en razones privadas no tendrían lugar en un espacio donde lo que se busca es determinar cuestiones de interés comunitario.

En los modelos de E-democracia este requisito no es tan claro. Al plantearse una Red de usuarios con la misma capacidad de influencia y participación, es probable que las intervenciones y los temas a tratar no sean razonables. Por ejemplo, imaginemos el caso de una sociedad intervenida por profundas doctrinas religiosas, donde los temas que se introducen en el debate se fundan en consideraciones sobre esas creencias (por ejemplo, la inmoralidad del matrimonio entre personas del mismo sexo)..$^{18}$

Resulta aun más problemático imaginarse las posibles resoluciones de esta situación en el marco de una E-democracia; las dos variantes que aparecen para imitar el filtro de razonabilidad propio de la democracia deliberativa son

17 Peña-Lopez, I. (2011) «Empowerment and Governance in the Information Society (II): digerati, goverati and the role of ICT4D»en JeDEM 3(1), pp. 1-21.

18 Sobre este tema ver: Abal, F. (2014 - en prensa) «Posibilidad de una Razón Publica Excluyente» en Ideas y Valores, Diciembre, № 156. 
(i) la intervención de los programadores o (ii) la exclusión de los temas que no promueven la participación de los usuarios.

El primero consistiría en un ordenamiento de los foros de discusión por parte del equipo técnico del soporte electrónico, el cual restringiría la introducción de temas fundados en consideraciones irrazonables al debate. Esta opción no es más que la continuación de los problemas democráticos actuales; al permitir que los programadores sean quienes determinen qué contenidos son razonables (aun si el equipo está conformado por distintas expresiones partidarias o independientes), habilitamos nuevamente la constitución de un estamento que determina sobre lo que puede discutirse.

El segundo es más complejo y tiene una vinculación más estrecha con la idea de razonabilidad. Una manera de excluir temas de los foros de deliberación electrónica seria diseñando una aplicación que diera de baja aquellos espacios de discusión que no motivan la participación de los demás usuarios. En algún sentido, es lo que ocurre en la democracia frente a aquellas reivindicaciones que no respetan el deber de reciprocidad. Sin embargo, existen muchos reclamos justos de minorías o sectores sociales excluidos que no generan el interés que debieran y bajo este tipo de dispositivo perderían un espacio importante. Por ejemplo, las constantes protestas de grupos aborígenes en Formosa (Argentina), que no parece interpelar a gran parte de la ciudadanía formoseña que continúa eligiendo a la misma fuerza política encargada de perseguirlos y atropellar sus derechos durante la última década.

\section{IV.2 IGUAL CONSIDERACIÓN DE TODOS LOS USUARIOS}

La democracia electrónica no permite hacer distinciones entre los usuarios. Esta caracteristica conlleva un problema teórico fundamental que, según creo, la democracia deliberativa está en mejores condiciones de enfrentar. La deliberación plantea una alternativa a los modelos de agregación de preferencias; se trata de crear mecanismos para que las personas construyan conjuntamente nociones propias del bien común y mejores medios para alcanzar el bienestar de la ciudadanía. En esta búsqueda es probable que deban hacerse distinciones entre los participantes del debate más afectados por la política X puesta en cuestión, y los menos afectados. Imaginemos una discusión como la ocurrida en Bolivia en el año 2012; el gobierno de Evo Morales propuso la construcción de una carretera que favorecería el comercio entre dos departamentos importantes del país (Beni y Cochabamba). Para ser construido debía desmontarse el TIPNIS (Territorio Indígena Parque Natural Isiboro-Secure); esta inciativa genero un inmediato repudio por parte de la comunidad aborigen local que reconocía ese espacio como propio y lo vinculaba con sus más profundas 
tradiciones cultuales..$^{19}$ La primera respuesta gubernamental fue llamar a un referéndum por la continuidad del proyecto. Considerando los hechos, y más allá del resultado final, una buena disposición deliberativa hubiera privilegiado los canales de expresión de aquellas personas que se veían más afectadas por la construcción de la carretera, en aquel caso las comunidades autóctonas.

Esta interpretación se funda en lo que considero es el núcleo de la deliberación; la construcción de una alternativa no oligárquica lleva a una ampliación de los espacios de toma de decisiones y en ellos la noción de bienestar general es construida entre todos los participantes. Si la cuestión es el bienestar, la manera más adecuada de proceder es considerando fundamentalmente las razones de quienes se verían gravemente afectados de tomarse o no la medida.

La E-democracia no presenta esta versatilidad y reconoce a todos los usuarios con la misma capacidad de influir en la toma de decisiones, cualquiera sea el tema en discusión. Si pudieran plantearse electrónicamente estas distinciones que volverían más sensibles las decisiones a tomar, solo podrían hacerse en función de una deliberación previa que las reconociera como relevantes; lo que muestra la incapacidad de las nuevas tecnologías para agotar las posibilidades de la deliberación real.

\section{IV.3 DESIDEOLOGIZACIÓN}

Los partidos piratas que promueven la E-democracia, surgen como una intervención política concreta de movimientos dispersos de usuarios cibernéticos, divulgadores de la ética hacker, a favor del uso de software libre y en contra de las leyes de copyright vigentes que consideraban obsoletas frente al vertiginoso desarrollo de las redes sociales y los grandes portales de información.

Sin embargo, su programa fue sumando otras reivindicaciones hasta consolidarse como una alternativa interesada en ofrecer mayores canales de participación ciudadana y procedimientos de toma de decisiones más eficientes y transparentes. En medio de la crisis europea, vienen logrando importantes resultados electorales, incorporando dos legisladores al parlamento europeo.

Rick Falkvinge, fundador del partido pirata sueco (tercera fuerza electoral en su país), y principal impulsor de esta nueva expresión, sostiene que no reproducen ninguna ideología existente.

«Tenemos gente de un extremo a otro, desde el anarquismo hasta el totalitarismo. No somos ni socialistas ni liberales. Somos piratas. Todos tienen las puertas abiertas. El único requisito es respetar los derechos humanos. Somos una ideo-

19 Para un resumen detallado del conflicto ,ver: $<$ http://codpi.org/observatorio/94-represion-gubernamental-a-la-marcha-por-el-tipnis $>$. 


\section{logía en nosotros mismos.» ${ }^{20}$}

Esta pequeña declaración de principios se expresa en las plataformas de los distintos partidos piratas con participación electoral, alrededor del mundo. El caso del Partido de la Red (Argentina) es un ejemplo de este tipo de construcción. En su propia página web encontramos la siguiente presentación:

«El Partido de la Red es un partido político que propone mejorar la democracia aprovechando las virtudes de Internet. En concreto, propone un modelo de participación ciudadana que es un híbrido de democracia directa y democracia representativa.» Acerca del PDR en http://partidodelared.org/acerca-del-pdr/

Los legisladores del Partido de la Red, de ser electos, se comprometen a votar en conformidad con lo que se decida entre todos los usuarios que libremente participen de una plataforma online (Democracia.OS). Este soporte muestra los principales proyectos de ley que se tratan en la legislatura porteña y permite a los ciudadanos participar de foros de discusión o delegar su voto en otro usuario que consideren con mayores conocimientos en el área (educación, salud, ambiente, cultura, etc.).

Podrían señalarse dos inconvenientes en este tipo de propuestas: (i) en primer lugar, el legislador pirata es simplemente un nexo entre los usuarios de la plataforma online y el voto, lo que le impide discutir libremente en la legislatura, sin antes haber sometido a la deliberación electrónica aquello puesto en discusión. Podría ser reemplazado por una computadora que mostrara los resultados de la votación previamente efectuada en el soporte online. (ii) El partido parece resuelto a seguir los distintos avatares de la realidad política en consonancia con lo que determinen los usuarios. Suponiendo que toda la ciudadanía participara de la plataforma online, sea cual sea el resultado (siempre que no promueva la violación de algún derecho humano), los legisladores piratas se ven compelidos a votar en conformidad. Esto establece un cierto vacío ideológico que parece reproducir una lógica mayoritarista, alejada de los valores que creemos fundamentales en la representación política. Cuando depositamos nuestro voto en un candidato, esperamos que lleve adelante una actividad legislativa conforme a ciertos principios no expuestos a los cambios coyunturales. La política argentina, por ejemplo, ha mostrado variados casos en los que la clase política ha adoptado actitudes demagógicas, para favorecer un aparente reclamo popular, que se han traducido en modificaciones legales discriminatorias e injustas. ${ }^{21}$

20 Entrevista concedida a Sergi Tarin del medio Publico.es, Link: < http://www.publico. es/270265/no-somos-ni-socialistas-ni-liberales-somos-piratas > (17/11/2009)

$21 \mathrm{Tal}$ es el caso de las llamada «leyes Blumberg», promovidas en el 2004 en Argentina, 


\section{CONCLUSIÓN}

Hasta aquí he mencionado algunos problemas (de implementación y teóricos) para reducir la deliberación real a las plataformas electrónicas. Sin embargo, es posible imaginar diseños institucionales en los cuales la aplicación de un modelo de E-democracia reducido podría resultar satisfactoria. Tal parece ser el caso de elecciones de autoridades administrativas en ámbitos universitarios o en asociaciones sin fines de lucro. En espacios reducidos, donde priman los intereses comunitarios y la consideración igualitaria de los afectados no requiere de modificaciones profundas en el debate, la construcción de plataformas online constituiría un medio de divulgación y de toma de decisiones más eficiente. Los acontecimientos que inauguran este artículo y las apreciaciones que he presentado, pretenden mostrar que no son éstas las condiciones de nuestras democracias actuales.

\section{REFERENCIAS BIBLIOGRÁFICAS}

Barabasi A. L. \& Albert R. (1999) «Emergence of scaling in random networks» en Science, vol. 286, pp. 509-511.

Barabasi A.L. (2001) «The physics of the Web» en Physics World, vol. 14/7, pp. 1-12.

Benhabib, S. (1999) «Models of Public Space: Hanna Arendt, the Liberal Tradition, and Jurgen Habermas» en Calhun, C., ed., Habermas and the Public Sphere. Massachusetts: MIT Press.

Bohman, J. (1996) Public Deliberation: Pluralism, Complexity, and Democracy. Cambridge: MIT Press.

Cohen, J. (2001) «Democracia y Libertad» en Elster, J., ed., La Democracia Deliberativa. Barcelona: Gedisa.

DAhlberG, L. (2001) «The Internet and democratic discourse» en Information, Communication \& Society 4:4, pp. 615-633.

Elster, J., editor (2001) La Democracia Deliberativa. Barcelona: Gedisa.

GAROT, M. J. (2006) «De la administración electrónica a una democracia digital»en Revista Direito $G V$, vol. 2, n 1 , pp. 89-11.

Guariglia, O. (2002) Una ética para el siglo XXI: ética y derechos humanos en un mundo postmetafisico. Bs. As. : FCE.

Habermas, J. (1999) «Popular Sovereignty as Procedure» en Bohman, J. y Rehg, W., ed., Deliberative democracy. Essays on reason and politics. Massachusetts: MIT Press.

ver: Tropea, F. (2007) «La ley 25.886 y la ruptura con los principios de la penalidad moderna» en Revista Electrónica Derecho Penal Online. Link < http://www.derechopenalonline.com/derecho. php?id=13,452,0,0,1,0>(30/11/2007) 
Marti, J. L Y CANALS, I. (2003) L’Àgora digital. Internet al servei de la participació ciutadana. Barcelona: Editorial Mediterrània - Fundació Catalunya Segle XXI.

Norris, P. Y Curtice, J. (2004) «If you build a political website, will they come?», Paper for APSA Committee for Political Sociology at the Annual Meeting of the American Political Science Association, September.

PeÑA-LoPeZ, I. (2011) «Empowerment and Governance in the Information Society (II): digerati, goverati and the role of ICT4D»en JeDEM 3(1); pp. 1-21.

PORRAS, J. I. (2005) «Internet y las nuevas oportunidades para la deliberación pública en los espacios locales» en Nueva Sociedad 195, enero-febrero, pp 102-116.

RoBles, J. M. Y GANuZA, E. (2011) «Internet y Deliberación. Dos ideas para comprender cómo afrontan los indignados la participación política» en Sociedad y Utopía 38: $243-262$.

Soderqvist, J. Y BARD, A. (2003) La netocracia. El nuevo poder en la red y la vida después del capitalismo. Madrid: Prentice Hall.

Federico Germán Abal. Universidad de Buenos Aires (UBA) - Facultad de Filosofía y Letras (FFyL) - Grupo de Filosofía Política (GFP)

Publicaciones recientes:

«Posibilidad de una Razón Publica Excluyente en Ideas y Valores» 63 (156), diciembre 2014, pp. 33-52.

Línea de investigación:

Área de trabajo: Filosofía Política y Ética

Dirección postal:

Ruiz de los Llanos 684 (departamento «d»), Ciudad Autónoma de Buenos Aires, Argentina, Código Postal: 1408

Dirección electrónica: Fede201011@live.com.ar 
